3 April 2009 version; Accepted by AJ

\title{
Astrometric Redshifts for Quasars
}

\author{
Michael C. Kaczmarczik, Gordon T. Richards, Sajjan S. Mehta \\ Department of Physics, Drexel University, Philadelphia, PA 19104 \\ David J. Schlegel \\ Lawrence Berkeley National Lab, 1 Cyclotron Road, MS 50R5032, Berkeley, CA 94720
}

\begin{abstract}
The wavelength dependence of atmospheric refraction causes differential chromatic refraction (DCR), whereby objects imaged at different optical/UV wavelengths are observed at slightly different positions in the plane of the detector. Strong spectral features induce changes in the effective wavelengths of broad-band filters that are capable of producing significant positional offsets with respect to standard DCR corrections. We examine such offsets for broad-emission-line (type 1) quasars from the Sloan Digital Sky Survey (SDSS) spanning $0<z<5$ and an airmass range of 1.0 to 1.8. These offsets are in good agreement with those predicted by convolving a composite quasar spectrum with the SDSS bandpasses as a function of redshift and airmass. This astrometric information can be used to break degeneracies in photometric redshifts of quasars (or other emission-line sources) and, for extreme cases, may be suitable for determining "astrometric redshifts". On the SDSS's southern equatorial stripe, where it is possible to average many multi-epoch measurements, more than $60 \%$ of quasars have emission-lineinduced astrometric offsets larger than the SDSS's relative astrometric errors of 25-35 mas. Folding these astrometric offsets into photometric redshift estimates yields an improvement of $9 \%$ within $\Delta z \pm 0.1$. Future multi-epoch synoptic surveys such as LSST and Pan-STARRS could benefit from intentionally making $\sim 10$ observations at relatively high airmass $(\mathrm{AM} \sim 1.4)$ in order to improve their photometric redshifts for quasars.
\end{abstract}

Subject headings: quasars: general — quasars: emission lines — galaxies: distances and redshifts - astrometry — atmospheric effects 


\section{Introduction}

The next generation of large-area survey facilities (e.g., Pan-STARRS, LSST, DES, VISTA/VST; Kaiser et al. 2002; Ivezić et al. 2008; The Dark Energy Survey Collaboration 2005; ; Arnaboldi et al. 2007) will image orders of magnitude more objects than it will be possible to obtain spectra for. As a result, robust determination of redshifts from photometric data is of crucial importance to these projects. While the distinctive " $4000 \AA$ break" facilitates photometric redshifts ("photo-z's") for galaxies (e.g., Connolly et al. 1995; Oyaizu et al. 2008), the majority of quasars $(z \lesssim 2.2$ ) lack such a strong spectral feature at observedframe optical wavelengths, making the determination of photometric redshifts for low- $z$ quasars more challenging. While it has been proven to be possible to determine photo- $z$ 's of quasars from the changes that their emission lines induce in their broad-band photometry (e.g., Wolf et al. 2001; Richards et al. 2001; Budavári et al. 2001; Weinstein et al. 2004; Ball et al. 2008) and from spectral energy distribution (SED) fitting (e.g., Brodwin et al. 2006; Rowan-Robinson et al. 2008), the accuracy of these photo-z's does not match that of galaxies and often suffers from catastrophic failures $(|\Delta z|>0.3)$.

Here we introduce a powerful new tool to aid redshift determination for emission-line objects (such as quasars) by taking advantage of the spectroscopic properties of the Earth's atmosphere. Light rays from extraterrestrial sources are bent according to Snell's law as they enter the Earth's atmosphere from the vacuum of space. As a result, except at the zenith, a celestial source observed from the Earth will appear higher in the sky than it actually is. The magnitude of this deflection depends on the index of refraction in air and the photon's angle of incidence. Since the index of refraction of air is a function of wavelength, light rays passing through the atmosphere undergo dispersion, whereby shorter wavelength photons are bent more than longer wavelength photons. Filippenko (1982) provides an in-depth discussion of this effect, reminding observers of the rather large magnitude of this effect even at moderate airmasses and the need to perform spectroscopy at the "parallactic angle".

This effect, known as differential chromatic refraction (DCR; e.g, Pier et al. 2003), is normally a source of nuisance for multi-band astrometry. Indeed oftentimes additional lenses are used to form an atmospheric dispersion corrector (ADC) to compensate for DCR in hardware, such as for the $2 \mathrm{dF}$ system (Lewis et al. 2002) on the Anglo-Australian Telescope. However, rather than being a nuisance, DCR can be used as a unique tool for estimating redshifts of emission-line objects. Software-based astrometric corrections for DCR are generally computed as a function of broad-band flux ratio (i.e., color), whereas the actual DCR in emission-line objects instead depends on the distribution of flux within the bandpass. The difference between the expected and observed astrometric displacements due to DCR enables determination of astrometric redshifts (or astro- $z$ 's) for strong emission-line objects. This 
discrepancy can be used separately from, or in conjunction with, more traditional photo- $z$ estimates to provide redshifts from imaging data. Indeed, contrary to conventional wisdom, the next generation of multi-epoch imaging surveys may want to perform a fraction of their observations at moderately high airmass in order to take full advantage of this effect.

The sections of this paper are as follows. Section 2 discusses DCR from a theoretical perspective, while $\S 3$ considers DCR observations from both single- and multi-epoch quasar data from the Sloan Digital Sky Survey (SDSS; York et al. 2000). Section 4 examines how these measurements can be used to improve photometric redshift estimation for quasars. Finally, $\S 5$ discusses some avenues for further work and $\S 6$ presents our conclusions.

\section{Differential Chromatic Refraction}

\subsection{Theory}

Differential chromatic refraction is a fact of life for any multi-band astronomical imaging survey. Because of the wavelength dependence of the refractive index of air, a source observed with a blue filter will appear slightly higher in the sky than the same source observed through a red filter. Figure 1 illustrates this effect. The left-hand panel indicates the magnitude of the effect as a function of wavelength; here for the SDSS filter set. The right-hand panel of Figure 1 illustrates why the blue image is higher in the sky than the red image. It is clear that refraction is a potentially large source of astrometric error and both it and DCR must be carefully corrected (either in hardware with an ADC or in software) in order to produce accurate astrometric solutions.

Here we explicitly consider the absolute theoretical wavelength depedence of the deflection of photons from astronomical sources. The angular deflection of an incoming photon by the Earth's atmosphere is given by

$$
R \simeq R_{0} \tan (Z),
$$

where $Z$ is the angle from the zenith (airmass, $\mathrm{AM}=\sec [Z]$; good for $Z<80$ ) and $R_{0}$ is determined from the index of refraction, $n$, as

$$
R_{0}=\frac{n^{2}-1}{2 n^{2}}
$$

(e.g., $\operatorname{Cox} 2000$ ). The wavelength dependence of the index of refraction of air can be described by

$$
[n(\lambda)-1] 10^{6}=64.328+\frac{29498.1}{146-(1 / \lambda)^{2}}+\frac{255.4}{41-(1 / \lambda)^{2}}
$$


where $\lambda$ is expressed in microns (e.g., Filippenko 1982). Thus, a flat-spectrum source 1 observed at $\mathrm{AM}=1.414(Z=45)$ in the SDSS $r$ band $(6165 \AA)$ will appear $57^{\prime \prime} .06$ higher in the sky (ignoring temperature, pressure, water vapor, and altitude effects) than it would in the absence of Earth's atmosphere; see Figure 1.

To determine the DCR for any given object, it is sufficient to know the effective wavelength of the object within a given bandpass. Following Schneider et al. (1983), effective wavelengths are given by

$$
\lambda_{e f f}=\bar{\lambda}_{j}(z)=\exp \frac{\int_{0}^{\infty} f_{\lambda(1+z)} S_{j}(\lambda) \ln (\lambda) d \lambda}{\int_{0}^{\infty} f_{\lambda(1+z)} S_{j}(\lambda) d \lambda} .
$$

The subscript $j$ represents a given bandpass, $u, g, r, i$ or $z$ (Fukugita et al. 1996). The incident flux at a given wavelength and redshift is indicated by $f_{\lambda(1+z)}$ while $S_{j}(\lambda)$ is the response of the bandpasse: 2 . The transmission curves, $S_{j}(\lambda)$, for the SDSS system include atmospheric extinction at the mean airmass of the SDSS survey, $\mathrm{AM}=1.3$. For a flat-spectrum source, the nominal effective wavelengths of the SDSS bandpasses are 3551, 4686, 6165, 7481, and $8931 \AA$, respectively for $u, g, r, i$, and $z$. For a power-law continuum with spectral index $\alpha_{\nu}=-0.5$, characteristic of quasars, the effective wavelengths are 3541, 4653, 6147, 7461, and 8904, respectively (Richards et al. 2001). Using these effective wavelengths, the DCR can be determined from Equations 1-3.

\subsection{DCR for Broad Emission-Line Quasars}

In reality, the effective wavelength will depend on the more complicated wavelength dependence for the SEDs of real astronomical sources. As broad-band photometry trades a loss of information (integration of a spectral energy distribution over $\sim 1000 \AA$ ) for a gain in signal-to-noise per unit time, the information from spectral features narrower than the photometric bandpasses is marginalized. Quasar emission lines, however, are sufficiently broad and strong that their effects are quite noticeable both on their colors (e.g., Cristiani \& Vio 1990; Richards et al. 2001), and their broad-band magnitudes (Richards et al. 2006). These emission lines can similarly shift the effective wavelength of a photometric bandpass. This shift occurs because the effective wavelength depends not only on the filter's transmission properties, but also on the distribution of the source's flux within the bandpass. Indeed, while

\footnotetext{
${ }^{1}$ We use the nomenclature $f_{\nu} \propto \nu^{\alpha}$, where a flat-spectrum source has $\alpha_{\nu}=0$.

${ }^{2}$ See http://www.sdss.org/dr6/instruments/imager/.
} 
astronomers are frequently tempted to assume a uniform transformation between broadband photometric systems, those transformations are, in fact, strongly dependent on the (observed frame) spectral energy distribution of the objects in question (e.g., Fukugita et al. 1996; Jester et al. 2005).

In Figure 2 we illustrate the effect of quasar emission lines on the effective wavelengths

within the SDSS broad-band photometric system. Shown is the Vanden Berk et al. (2001) SDSS composite quasar spectrum at four redshifts where the Ly $\alpha$ or C IV emission lines lie at the red or blue edge of the $u$ filter. Figure 3 shows the full redshift dependence of the effective wavelength for the mean quasar; here we have convolved the mean quasar spectrum with the SDSS bandpasses according to Equation 4. Compared to a power-law continuum source, the effective wavelength of the SDSS bandpasses can change by as much as $150 \AA$ for quasars. (For $z \lesssim 0.3$ and $z \gtrsim 3.25$ the Vanden Berk et al. 2001 composite spectrum does not fully span the $z$ and $u$ bands, respectively and the expected changes in effective wavelength cannot be determined from the composite quasar spectrum.) We can use these effective wavelengths to determine the magnitude of the DCR for emission-line objects as a function of redshift, but first we need to understand how DCR corrections are applied, particularly in the case of SDSS astrometry.

\subsection{SDSS Astrometry}

As can be seen from Figure1, if no DCR corrections were applied, then potentially large positional differences between bandpasses would severly complicate multi-color photometry. Since the true SED of an object is unknown, the broad-band photometry itself provides the best model for the wavelength dependence of the SED, which is needed to determine the DCR correction. In practice, this does not involve making a full correction based on a theoretical model, but rather by including a color-dependent term in the astrometric solution (e.g., Pier et al. 2003). For SDSS imaging, the DCR correction is modeled as a linear function of color for stars bluer than $u-g=3$ in the $u$-band and $g-r=1.5$ in the $g$-band; redder stars are modeled with constant color. For the $r, i$, and $z$ bands, the DCR is taken to be a linear function of the $r-i$ color for all stars. For more details, see $\S 5.3$ in Pier et al. (2003) 3 . This color-dependent modeling of the DCR means that, for purely power-law sources, the positional agreement between the five astrometrically-calibrated SDSS bandpasses should not show any evidence of DCR.

\footnotetext{
${ }^{3}$ Note that the color dependence was misstated in Pier et al. (2003) and is corrected at http://www.sdss.org/dr7/products/general/astrometry.html
} 
Any residual astrometric offsets will be due to the difference between the color-dependent correction that is applied to the SDSS astrometry and the actual DCR for any particular object, which depends on the distribution of flux within a bandpass rather than the distribution of flux across bandpasses (i.e., the color). As such, for our purposes, we can ignore any altitude, temperature, pressure, or water vapor corrections to the index of refraction of air since we are only concerned with the relative DCR with respect to a presumed model.

\subsection{Predicting SDSS Quasar Positions}

As a result of the broad-band color-dependent DCR correction, we cannot simply use the effective wavelengths from the composite quasar spectrum to determine the expected positional offsets of quasars. Rather, we must compare these offsets with those expected from the astrometric model. For our analysis, we will consider two models for DCR. One is based on synthetic colors determined from the composite quasar spectrum (e.g., Richards et al. 2001), the other is based on the mean power-law SED of quasars, $\alpha_{\nu} \sim-0.5$. Accordingly, we modify Equation 1 to

$$
R \simeq\left[R_{0}(\mathrm{SED})-R_{0}(\text { color })\right] \tan (Z)
$$

or

$$
R \simeq\left[R_{0}(\mathrm{SED})-R_{0}(\mathrm{PL})\right] \tan (Z)
$$

Once we have determined $\lambda_{\text {eff }}$ (and thus $R_{0}$ ) for both the composite spectrum and the model in each bandpass as a function of redshift, positional offsets can be calculated for any airmass according using Equation 5. Figure 4 shows the predicted offsets for the mean quasar as a function of redshift and airmass in each of the five SDSS bandpasses. Here we make no airmass corrections to the SDSS bandpasses despite the fact that they are given specifically for $\mathrm{AM}=1.3$. As demonstrated by D. Schlegel (unpublished), the 25-35 mas

rms astrometric errors between bandpasses of the SDSS imaging survey (Pier et al. 2003) are comparable to or less than the astrometric offsets due to quasar emission lines redshifting through the SDSS broad-band filters.

Looking ahead to comparisons with the data, it must be realized that the DCR effect is entirely in the direction along the "parallactic angle" (or perpendicular to the horizon), thus we adopt the notation $R_{\|}$in Figure 4 to indicate positional offsets in the direction where DCR is applicable. Our sign convention is that bluer effective wavelengths result in images higher in the sky than expected, yielding positive astrometric offsets.

We will see in $\S 3$ that the positions of SDSS objects in the ugiz bands are given 
with respect to the $r$ band, which defines the SDSS astrometric system. That is the $r$ band positional offsets do not agree with Figure 4, but rather are all identically zero (by definition). Thus it will often be more convenient to examine the relative changes in position between two bandpasses than the absolute deviation for a single bandpass. The difference in positional offsets between two adjacent colors, $\Delta R_{\|, m-n}$ is just $R_{\|, m}-R_{\|, n}$, where $m$ and $n$ represent the two bandpasses and. Figure 5 shows the resulting $\Delta R_{\|, m-n}$ relations as a function of redshift for adjacent bandpasses at various airmasses. At sufficiently high airmass or by averaging multi-epoch observations at low airmass, these positional offsets can exceed the astrometric errors and can be used as quasar redshift diagnostics.

\section{Observations}

\subsection{Sloan Digital Sky Survey Data}

While the DCR effect is a well-known phenomenon in astrometry and is generic to all ground-based data sets, our application of it to astrometric redshifts is based on data from the Sloan Digital Sky Survey (SDSS). The SDSS photometric data is summarized in a series of papers including Gunn et al. (1998), York et al. (2000), Hogg et al. (2001), Smith et al. (2002), Ivezić et al. (2004), Gunn et al. (2006), and Tucker et al. (2006). In $\S$ 3.3, we will make particular use of the data from SDSS "Stripe 82" (Annis et al. 2006), where multiple epochs of SDSS imaging data have been combined, reducing both the photometric and astrometric errors. In addition, we will make use of spectroscopically-confirmed SDSS quasars that were selected according to Richards et al. (2002) and tiled according to Blanton et al. (2003). Discussions of the SDSS filter system and astrometric solutions can be found in Fukugita et al. (1996), Stoughton et al. (2002), Pier et al. (2003), and also at http://www.sdss.org/dr7/algorithms/astrometry.html.

\subsection{Single-Epoch Data}

We now compare our theoretical predictions with actual measurements for the 77,000 quasars from the DR5 quasar catalog (Schneider et al. 2007). SDSS astrometry is reported with respect to the $r$ band (corrected to $A M=1$ ). The positions of the quasars in the other bandpasses were extracted from the photoObjAll in the SDSS database 4 . These values are given in the database as offsetRA $\left(=\mathrm{RA}_{x}-\mathrm{RA}_{r} \cos \left[\mathrm{Dec}_{r}\right]\right)$ and offsetDec $\left(=\operatorname{Dec}_{x}-\mathrm{Dec}_{r}\right)$,

\footnotetext{
${ }^{4}$ http://cas.sdss.org/dr6/
} 
where $x$ represents one of the ugriz bandpasses. By definition offsetRA $\mathrm{f}_{r}$ and off $\mathrm{setDec}_{r}$ are identically zero as the $r$ band (corrected for atmospheric refraction) defines the SDSS astrometric system.

The DR5 quasar catalog spans a redshift range of $0.08 \leq \mathrm{z} \leq 5.4$ and an airmass range of $1.002 \leq \mathrm{AM} \leq 1.795$. As we are interested only in the positional offsets due to DCR, we must first determine the parallactic angle for each of the observations and project the observed offsets onto it. The parallactic angle, $p$, is given by

$$
\sin (p)=\cos (\phi) \frac{\sin \mathrm{HA}}{\sin (Z)},
$$

where $\phi$ is the latitude of the observatory and HA is the "hour angle" of the observation

(e.g., Filippenko 1982). That portion of the positional offsets parallel to the parallactic angle comes from a combination of astrometric errors and atmospheric dispersion, while the portion perpendicular to the parallactic angle is due only to astrometric errors.

Figure 6] shows the resulting $\Delta R_{\|, m-n}$ for the SDSS ugr bandpasses. While the deviation of the astrometric offsets from the model is more noticeable at higher airmass and most SDSS observations are performed at the minimum possible airmass, the effect is still quite apparent in the ensemble average.

\subsection{Multi-Epoch Data}

Although Figure 6] shows that emission-line-induced astrometric offsets for quasars are evident in single-epoch SDSS data, they are not significantly larger than the mean astrometric errors for individual observations, reducing the utility of the astrometric offsets for redshift determination. However, for the SDSS southern equatorial region (aka, "stripe 82") and for the upcoming multi-epoch synoptic surveys (e.g., Pan-STARRS and LSST), it is possible to combine data from different epochs in order to reduce random astrometric errors and increase the signal-to-noise of the astrometric offsets along the parallactic angle.

To illustrate this improvement we have queried the SDSS stripe 82 database for multiple observations of objects. While stripe 82 has dozens of repeat scans, not all of these observations were observed at airmass high enough to show the DCR effect and not all were observed at the same airmass. Thus, we only used observations objects with AM $\geq 1.2$ and we have combined multiple such observations together, scaling by $\tan (Z)$ to a mean airmass of 1.3. This approach allows us to show not only the benefits of combining the few dozen epochs available from SDSS stripe 82 data, but also the hundreds of epochs that will be available in future imaging surveys. 
Figure 7 shows the resulting, more accurate, positional offsets in the $u$, and $g$ bandpasses, along with their difference for 6430 quasars in SDSS stripe 82. Comparison of the bottom panel of Figure 7 and the top panel of Figure 6 demonstrates that combining multiple observations has significantly reduced the positional scatter. The range of astrometric offsets is nearly 300 mas (e.g., $z \sim 1.7$ vs. $z \sim 2.1$ ), as compared with the $25-35$ mas astrometric errors for the single-epoch SDSS data. While few objects have offsets considerably larger than 30 mas, over $60 \%$ of the quasars have astrometric offsets at least this large. Moreover, by reducing random scatter with combined multi-epoch data, astrometric offsets smaller than 30 mas carry useful information. For example a measured value of $\Delta R_{\|, u-g}=0$ may not uniquely identify the redshift, but it does suggest redshift ranges that are higly unlikely.

Figure 8 illustrates the reduction of random astrometric errors from 25-35 mas in single epoch observations to under 10 mas after a few dozen combined observations. To determine the astrometric improvement we took the $u$ and $g$ band offsets perpendicular to the parallactic angle for 50 hot white dwarf observations. That is we looked at the astrometric offsets in the direction where we expect only random errors and not systematic DCR effects. Those observations were bootstrap resampled leaving out $\mathrm{N}$ objects at a time from 1 to 50 in order to determine the mean improvement as a function of the number of epochs combined. While individual observations are noisy, the mean offets in the direction parallel to the parallactic angle (due to both signal and noise) are much larger than the perpendicular scatter (due to noise only). These differences bode well for the inclusion of astrometric offsets in photometric redshift estimation and, perhaps, in object classification (e.g., see the object classification work by Lang et al. 2008 based on proper motions of objects in stripe 82).

\section{Astrometric Redshifts}

Since the random astrometric errors for the combined multi-epoch data are less than the range of astrometric offsets for quasars, these values can aid in redshift estimation. Figure 9 illustrates how DCR can be helpful in this endeavor. For example, emission features cause a blueward dip in the $u-g$ color for quasars with $1.7<z<2.1$. The $u-g$ color is roughly degenerate for a quasar at $z=1.7$ and $z=2.1$, which is a source of catastrophic error for

normal photo- $z$ 's. The color-coding of Figure 9] shows that including the $u-g$ astrometric offset breaks this degeracy by identifying the color of the $z=1.7$ quasars as being due to the emission feature on the blue side of the bandpass rather than on the red side of the bandpass.

Because the astrometric offsets are roughly Gaussian distributed (as are quasar colors), it is straightforward to include such measurements in our existing algorithm for redshift 
estimation as discussed in Weinstein et al. (2004). We can simply treat the observed $R_{\|, u}$ and $R_{\|, g}$ measurements as additional "colors". This method compares each observed color to the mean (and standard deviation) color at every redshift, choosing the redshift that minimizes the $\chi^{2}$.

For the 6430 quasars from stripe 82 we have been able to combine multiple observations, reducing both the photometric and astrometric errors. To determine the photo- $z$ improvement due to our astro- $z$ 's, we must first determine the photo- $z$ distribution using the improved photometry of these objects since we want to distinguish between astrometric and photometric improvements. Photo-z's for these 6430 quasars are first computed using only the five SDSS magnitudes (and errors). We perform 5-fold cross-validation whereby $80 \%$ of the sample is used for training and $20 \%$ is used for testing. Five iterations are used so that photo-z's are estimated for the full sample. These 4-color photo- $z$ 's provide a baseline for comparison with redshift estimates that also include astrometric offsets.

We then repeat this process, now adding the $R_{\|, u}$ and $R_{\|, g}$ measurements, treating them as the 5th and 6th colors. Any improvement over the 4-color photo- $z$ 's must be due to the added astrometric information. Figure 10 compares the spectropic versus photometric (and photometric+astrometric) redshifts for the full sample. Objects appearing along the diagonal have accruately estimated redshifts, whereas off-diagonal objects indicate catastrophic photometric redshift errors (Richards et al. 2001). While there are still errors in the photometric+astrometric sample, it can be seen that inclusion of astrometric information results in somewhat fewer catastropic errors and a tighter distribution along the diagonal (i.e., the precision of those objects with accurate photometric redshifts has been improved). The improvement can be better seen when plotting the fractional redshift error as shown in Figure 11. Adding astrometric redshifts reduces the fractional error from $5 \%$ to $3 \%$ (excluding catastrophic errors). It also increases the fraction of objects within $\Delta z \pm 0.3$ by $3 \%$ and the fraction within $\Delta z \pm 0.1$ by $9 \%$.

We emphasize that this improvement comes simply from treating the residual astrometric offsets as colors using our existing photo- $z$ algorithm. In reality, it would make more sense to use the offsets in a different manner. In particular, in addition to reducing the scatter in quasar redshift estimates, Figure 9] suggests that astro-z's can help break color degeneracies and will help improve redshift estimates for objects with catastropic errors. For example, broad absorption line quasars (e.g., Trump et al. 2006) and dust reddened quasars (Richards et al. 2003; Maddox et al. 2008) can ruin color-based photometric redshifts algorithms. High-redshift quasars become very red in $u-g$ as a result of Lyman- $\alpha$ forest absorption, but intrinsically blue quasars that are reddened by dust can appear just as red, which results in catastrophic photo- $z$ errors. However, the atmospheric dispersion effect only 
depends on the distribution of flux within the bandpass. As such, a moderate amount of dust has little effect on the positional offsets due to strong emission lines. This suggests that astro- $z$ 's may perform better when used as priors in advance of a standard photo- $z$ algorithm.

\section{Future Work}

\subsection{Other Emission-Line Objects}

We have concentrated on the applications of astrometric offsets specifically for quasars; however, the method is potentially applicable to other emission-line objects (e.g., type 2 quasars and supernovae). For type 2 quasars the emission lines are narrow, but their strength means that we can expect to see measurable astrometric offsets that are a function of redshift. Using a template type 2 quasar spectrum (e.g., Zakamska et al. 2003), we can estimate the astrometric offsets due to strong emission lines in type 2 quasar spectra. Figure 12 shows that, over the range $0.05<z<0.4, R_{\|, u-g}$ falls by almost 90 mas, while $R_{\|, u-g}$ rises by 75 mas. Thus redshifts for type 2 quasars with $z \leq 0.4$ could be distinguished at roughly the same level of accuracy as type 1 quasars.

It may also be possible to estimate redshifts for supernovae using astrometric offsets. While their SEDs are highly time-dependent and it would not be possible to combine multiple epochs, if the distinctive emission features at each epoch produced redshifts estimates that were in agreement, these astro-z's might be as reliable as they would be if the SEDs were not time-dependent. Most SNe spectral templates don't cover enough of the UV to properly estimate their positional offsets with redshift, but based on the existing coverage longward of $3000 \AA$, it would appear that the range of $u$ - and $g$-band offsets is as large as $0{ }^{\prime \prime} 15$ - certainly enough to make an initial guess at the redshift.

\subsection{Object Classification}

Just as the nature of the atmospheric offsets allows improvements in redshift estimation, the offsets could also be utilized in object classification itself. While astrometric solutions attempt to remove DCR, they can only do so at the level of broad-band photometric accuracy, whereas DCR is sensitive to features smaller than the width of the photometric bandpasses. As such, objects with distinctive SEDs may stand out enough in astrometric offset space to aid in object classification. While astrometric offsets alone are not likely to be useful for object classification, coupling these astrometric measurements with color and variability 
information within the context of modern Bayesian classification algorithms (Richards et al. 2009) could prove very fruitful in terms of the accuracy of object classification from broadband imaging data.

\subsection{Upcoming Imaging-only Surveys}

While most surveys aim to obtain observations as close to the meridian as possible (and thus at the lowest airmass possible), any object with declination different from the latitude of the observatory will necessarily be observed at AM $>1$. Thus any imaging survey will naturally yield observations that can be used with our approach. As refraction increases with airmass, one should ask whether future surveys may wish to obtain a fraction of their observations at relatively high airmass. Such observations will result in a loss of photons (particularly in the ultraviolet), so one must consider the details of the tradeoff between low and high airmass observations.

We have already shown in Figure 8 that combining just a few observations can go a long way towards reducing random astrometric errors below the level of anomalous DCR offsets for quasars. We next ask whether there is utility in making observations at very high airmass in order to increase the snr of the anomalous DCR effect. In Figure 13 we overplot the expected offsets for quasars in both the $u$ - and $g$-bands for airmasses 1.1, 1.4, and 1.8. It can be seen that an airmass of 1.1 is not sufficient for breaking redshift degeneracies, which implies that some level of concerted effort will be needed to obtain observations off of the meridian. At an airmass of 1.4, on the other hand, there is sufficient range to clearly indentify certain redshifts, even considering the scatter of the measurements as compared to these theoretical distributions. Extending to $\mathrm{AM}=1.8$, however, seems to offer little improvement, perhaps with the exception of the $z \sim 2.1$ and $z \sim 3.3$.

A full cost-benefit analysis of the trade-offs between more observations and higher airmass is beyond the scope of this paper as such analysis is highly system dependent, but it would seem that of order 10 observations at a moderate airmass of $\sim 1.4$ would be sufficient to yield significant improvement in photometric redshifts for quasars without causing significant problems for observational cadence or data reduction.

While the gains in photometric redshift accuracy due to the addition of astrometric information shown herein are modest $(\sim 9 \%)$, they undersell the utility of astrometric redshifts for the next generation of imaging surveys which will be able to combine hundreds instead of tens of epochs of data, each epoch with astrometric accuracy superior to that from the SDSS. For example, at $r=22$ the LSST astrometric errors are expected to be $\sim 15$ mas and 
there will exist over 200 epochs of data (Ivezić et al. 2008).

\section{Conclusions}

Astrometrists have long been dealing with the dispersive nature of our atmosphere when performing broad-band photometry and astrometry. Here we show that the dispersive nature of our atmosphere can actually be a boon rather than a burden. Objects with strong emission lines produce measurable astrometric offsets (with respect to an expected model) in the ensemble average even when observed at relatively low airmass. The signal-to-noise of the effect can be maximized by either combining many observations at low airmass or fewer observations at higher airmass. With well-measured astrometric offsets, it is possible to both break degeneracies in photometric redshift estimates of quasars and to reduce their overall scatter. For combined, multi-epoch data from the SDSS stripe 82 region, adding astrometric information increases the fraction of quasars with correct photometric redshifts by as much as $9 \%(\Delta z \pm 0.1)$. Given the lack of spectroscopic capabilities for future imaging surveys, the community would do well to build astrometric redshifts into their survey planning.

GTR acknowledges support from an Alfred P. Sloan Research Fellowship. We thank Željko Ivezić, Jeff Munn, and Don Schneider for comments on the manuscript and Bob Hindsley for early work on DCR in the SDSS astrometric system. Funding for the SDSS and SDSS-II has been provided by the Alfred P. Sloan Foundation, the Participating Institutions, the National Science Foundation, the U.S. Department of Energy, the National Aeronautics and Space Administration, the Japanese Monbukagakusho, the Max Planck Society, and the Higher Education Funding Council for England. The SDSS is managed by the Astrophysical Research Consortium for the Participating Institutions. The Participating Institutions are the American Museum of Natural History, Astrophysical Institute Potsdam, University of Basel, Cambridge University, Case Western Reserve University, University of Chicago, Drexel University, Fermilab, the Institute for Advanced Study, the Japan Participation Group, Johns Hopkins University, the Joint Institute for Nuclear Astrophysics, the Kavli Institute for Particle Astrophysics and Cosmology, the Korean Scientist Group, the Chinese Academy of Sciences (LAMOST), Los Alamos National Laboratory, the MaxPlanck-Institute for Astronomy (MPIA), the Max-Planck-Institute for Astrophysics (MPA), New Mexico State University, Ohio State University, University of Pittsburgh, University of Portsmouth, Princeton University, the United States Naval Observatory, and the University of Washington. 


\section{REFERENCES}

Annis, J. T., et al. 2006, in BAAS, vol. 38 of $B A A S, 1197$

Arnaboldi, M., Neeser, M. J., Parker, L. C., Rosati, P., Lombardi, M., Dietrich, J. P., \& Hummel, W. 2007, The Messenger, 127, 28

Ball, N. M., Brunner, R. J., Myers, A. D., Strand, N. E., Alberts, S. L., \& Tcheng, D. 2008, ApJ, 683, 12

Blanton, M. R., Lin, H., Lupton, R. H., Maley, F. M., Young, N., Zehavi, I., \& Loveday, J. 2003, AJ, 125, 2276

Brodwin, M., et al. 2006, ApJ, 651, 791, arXiv:astro-ph/0607450

Budavári, T., et al. 2001, AJ, 122, 1163, arXiv:astro-ph/0106036

Connolly, A. J., Csabai, I., Szalay, A. S., Koo, D. C., Kron, R. G., \& Munn, J. A. 1995, AJ, 110, 2655, arXiv:astro-ph/9508100

Cox, A. N. 2000, Allen's astrophysical quantities (Allen's Astrophysical Quantities)

Cristiani, S. \& Vio, R. 1990, A\&A, 227, 385

Filippenko, A. V. 1982, PASP, 94, 715

Fukugita, M., Ichikawa, T., Gunn, J. E., Doi, M., Shimasaku, K., \& Schneider, D. P. 1996, AJ, 111, 1748

Gunn, J. E., et al. 1998, AJ, 116, 3040

- 2006, AJ, 131, 2332, arXiv:astro-ph/0602326

Hogg, D. W., Finkbeiner, D. P., Schlegel, D. J., \& Gunn, J. E. 2001, AJ, 122, 2129

Ivezić, v., Tyson, J. A., Allsman, R., Andrew, J., Angel, R., \& for the LSST Collaboration 2008, ArXiv e-prints, 805, 0805.2366

Ivezić, Ž., et al. 2004, Astronomische Nachrichten, 325, 583, arXiv:astro-ph/0410195

Jester, S., et al. 2005, AJ, 130, 873, arXiv:astro-ph/0506022

Kaiser, N., et al. 2002, in Survey and Other Telescope Technologies and Discoveries. Edited by Tyson, J. Anthony; Wolff, Sidney. Proceedings of the SPIE, Volume 4836, pp. 154-164 (2002)., eds. J. A. Tyson \& S. Wolff, vol. 4836 of Presented at the Society of Photo-Optical Instrumentation Engineers (SPIE) Conference, 154-164 
Lang, D., Hogg, D. W., Jester, S., \& Rix, H.-W. 2008, ArXiv e-prints, 808, 0808.4004

Lewis, I. J., et al. 2002, MNRAS, 333, 279

Maddox, N., Hewett, P. C., Warren, S. J., \& Croom, S. M. 2008, MNRAS, 386, 1605, arXiv:0802.3650

Oyaizu, H., Lima, M., Cunha, C. E., Lin, H., Frieman, J., \& Sheldon, E. S. 2008, ApJ, 674, 768, arXiv:0708.0030

Pier, J. R., Munn, J. A., Hindsley, R. B., Hennessy, G. S., Kent, S. M., Lupton, R. H., \& Ivezić, Ž. 2003, AJ, 125, 1559

Richards, G. T., et al. 2001, AJ, 121, 2308

Richards, G. T., et al. 2001, AJ, 122, 1151, arXiv:astro-ph/0106038

Richards, G. T., et al. 2002, AJ, 123, 2945

Richards, G. T., et al. 2003, AJ, 126, 1131, arXiv:astro-ph/0305305

Richards, G. T., et al. 2006, AJ, 131, 2766

Richards, G. T., et al. 2009, ApJS, 180, 67, 0809.3952

Rowan-Robinson, M., et al. 2008, MNRAS, 386, 697, arXiv:0802.1890

Schneider, D. P., Gunn, J. E., \& Hoessel, J. G. 1983, ApJ, 264, 337

Schneider, D. P., et al. 2007, AJ, 134, 102, arXiv:0704.0806

Smith, J. A., et al. 2002, AJ, 123, 2121

Stoughton, C., et al. 2002, AJ, 123, 485

The Dark Energy Survey Collaboration 2005, ArXiv Astrophysics e-prints, astro-ph/0510346

Trump, J. R., et al. 2006, ApJS, 165, 1, arXiv:astro-ph/0603070

Tucker, D. L., et al. 2006, Astronomische Nachrichten, 327, 821, arXiv:astro-ph/0608575

Vanden Berk, D. E., et al. 2001, AJ, 122, 549

Weinstein, M. A., et al. 2004, ApJS, 155, 243, arXiv:astro-ph/0408504 
Wolf, C., Meisenheimer, K., \& Röser, H.-J. 2001, A\&A, 365, 660, arXiv:astro-ph/0010092

York, D. G., et al. 2000, AJ, 120, 1579

Zakamska, N. L., et al. 2003, AJ, 126, 2125, arXiv:astro-ph/0309551 

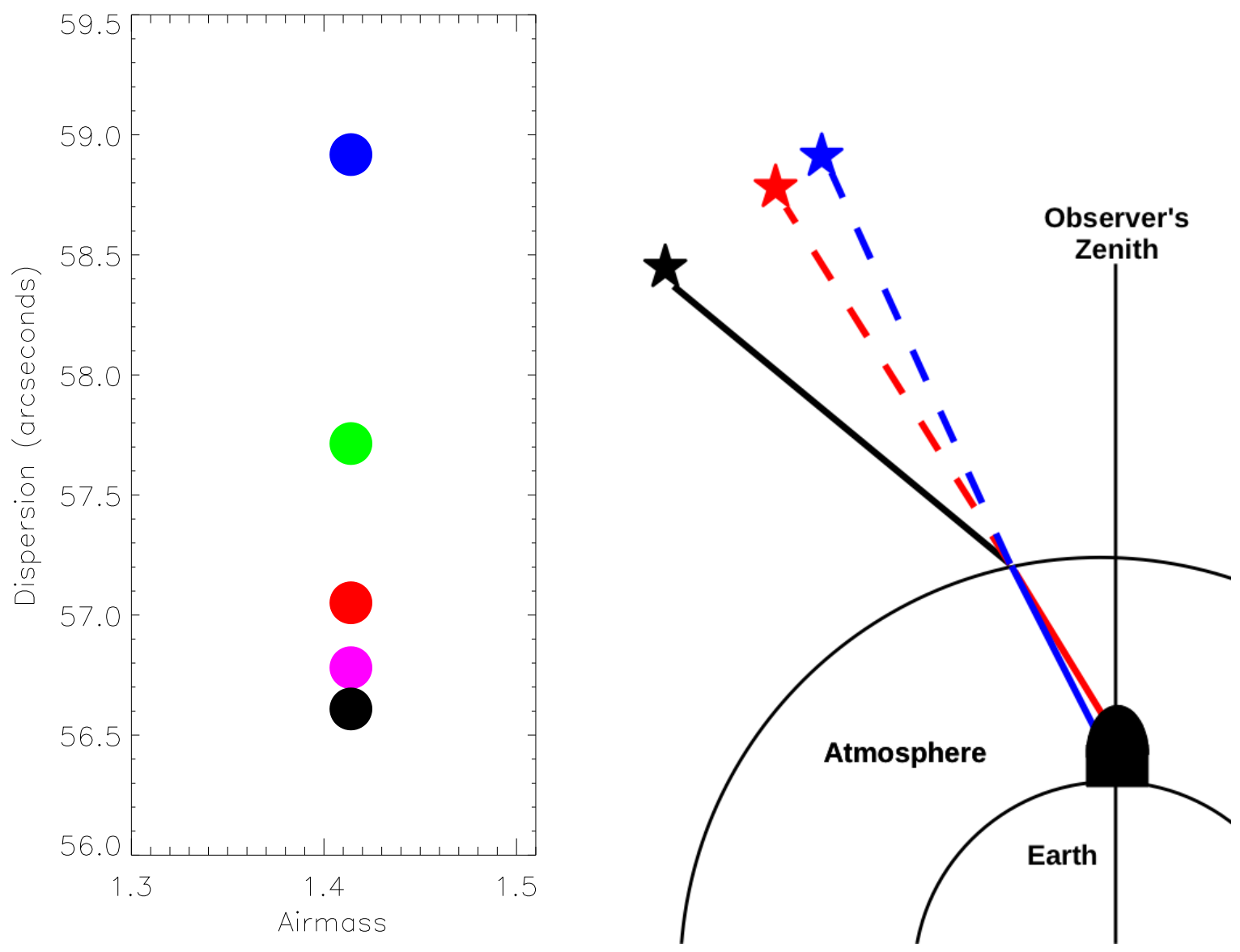

Fig. 1.- Left: Differential chromatic refraction (DCR) for a flat-spectrum object observed in the SDSS photometric system at a zenith angle of 45 degrees $(\mathrm{AM}=1.414)$. The color coding is $u$ =blue, $g$ =green, $r=$ red, $i=$ magenta, $z=$ black. Objects appear higher in the sky when observed in blue bandpasses than in red bandpasses. Right: DCR schematic example. The solid black line indicates the incoming multi-chromatic light rays. The solid red and blue lines indicate the differential chromatic refraction of the incoming beam, with blue light rays being bent more than red. The dashed blue and red lines indicate the apparent location on the sky of the object as seen by the blue and red filters. 

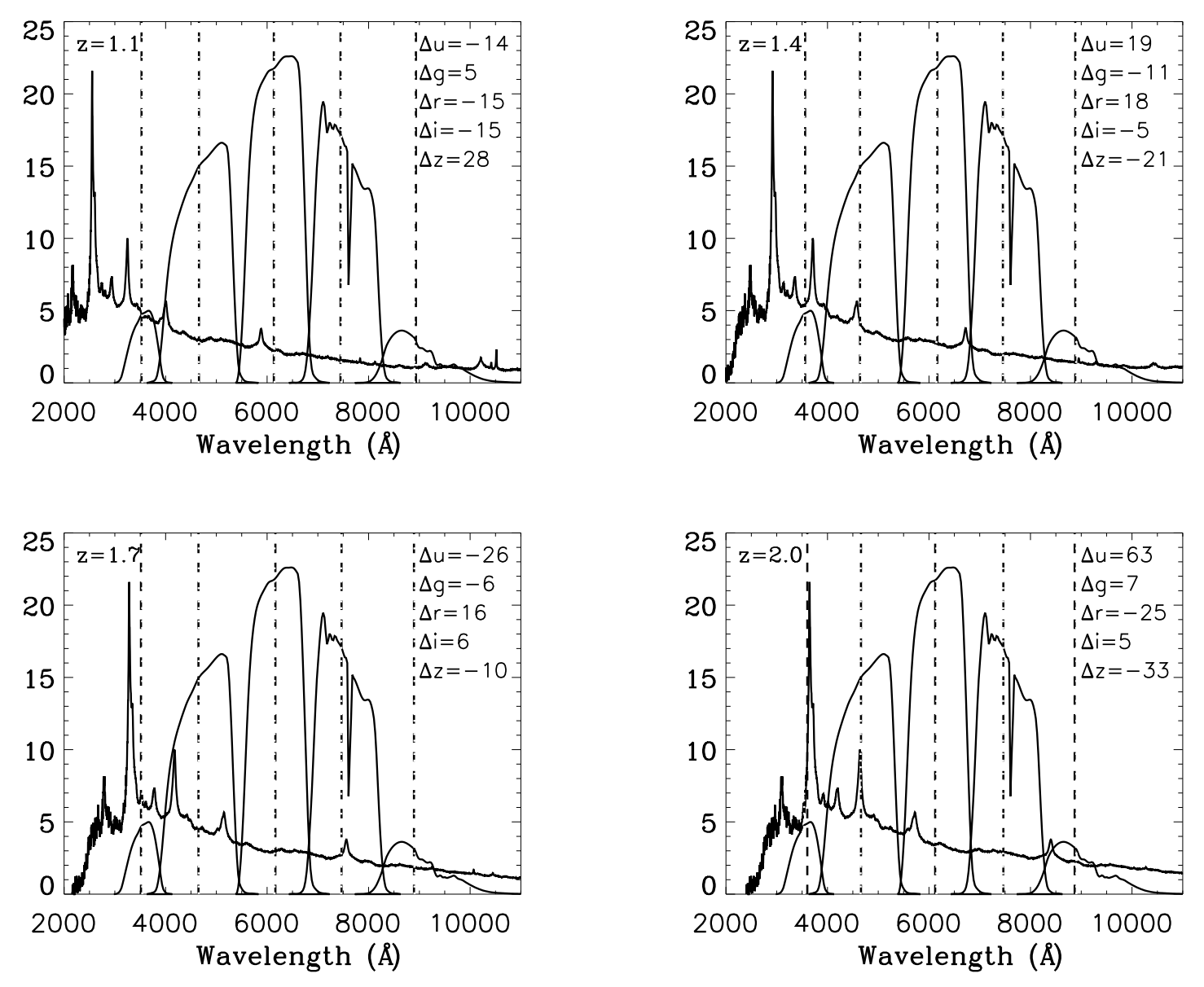

Fig. 2.- Change in effective wavelength as a function of redshift for a composite quasar SED (Vanden Berk et al. 2001) as compared to a power-law continuum source. The panels show quasars at redshifts 1.1, 1.4,1.7, and 2.0, illustrating the effect of strong lines moving from the blue to the red side of a bandpass (e.g., C IV in $g$ for $z=1.1$ and 1.4 and Lyman- $\alpha$ in $u$ for $z=1.7$ and 2.0). In the upper right-hand corner of each panel, the wavelength offset (in Angstroms) with respect to the nominal effective wavelength is given for each bandpass. 


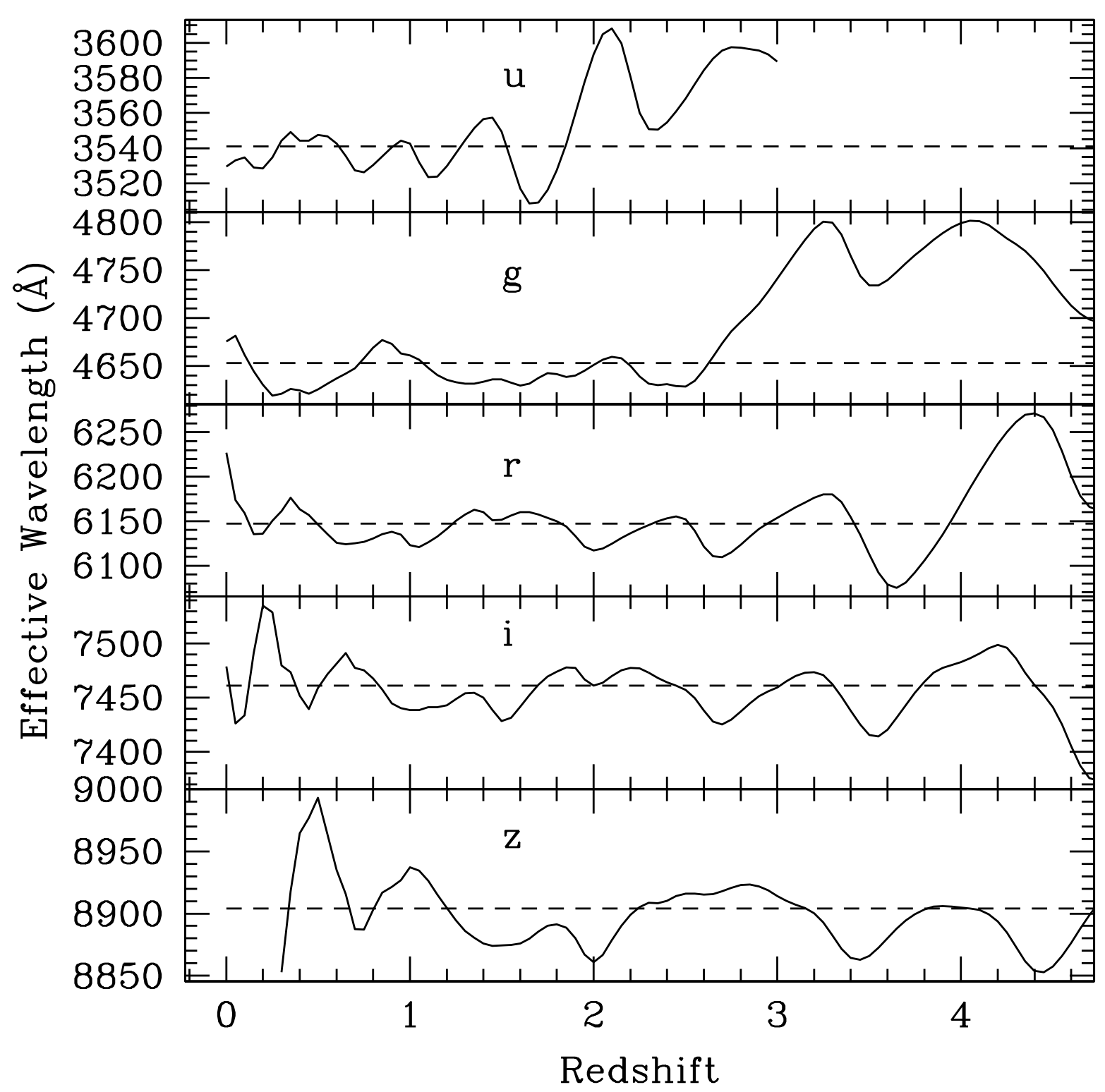

Fig. 3.- Expected change in effective wavelength for a quasar SED as a function of redshift. As a strong emission line passes through a given filter, the effective wavelength first shifts to the blue, then to the red, then returns to the standard value as the line exits the filter. Each panel shows one of the ugriz filters and its nominal expected wavelength (dashed) as compared with the predicted expected wavelength for a composite quasar spectrum (solid). 


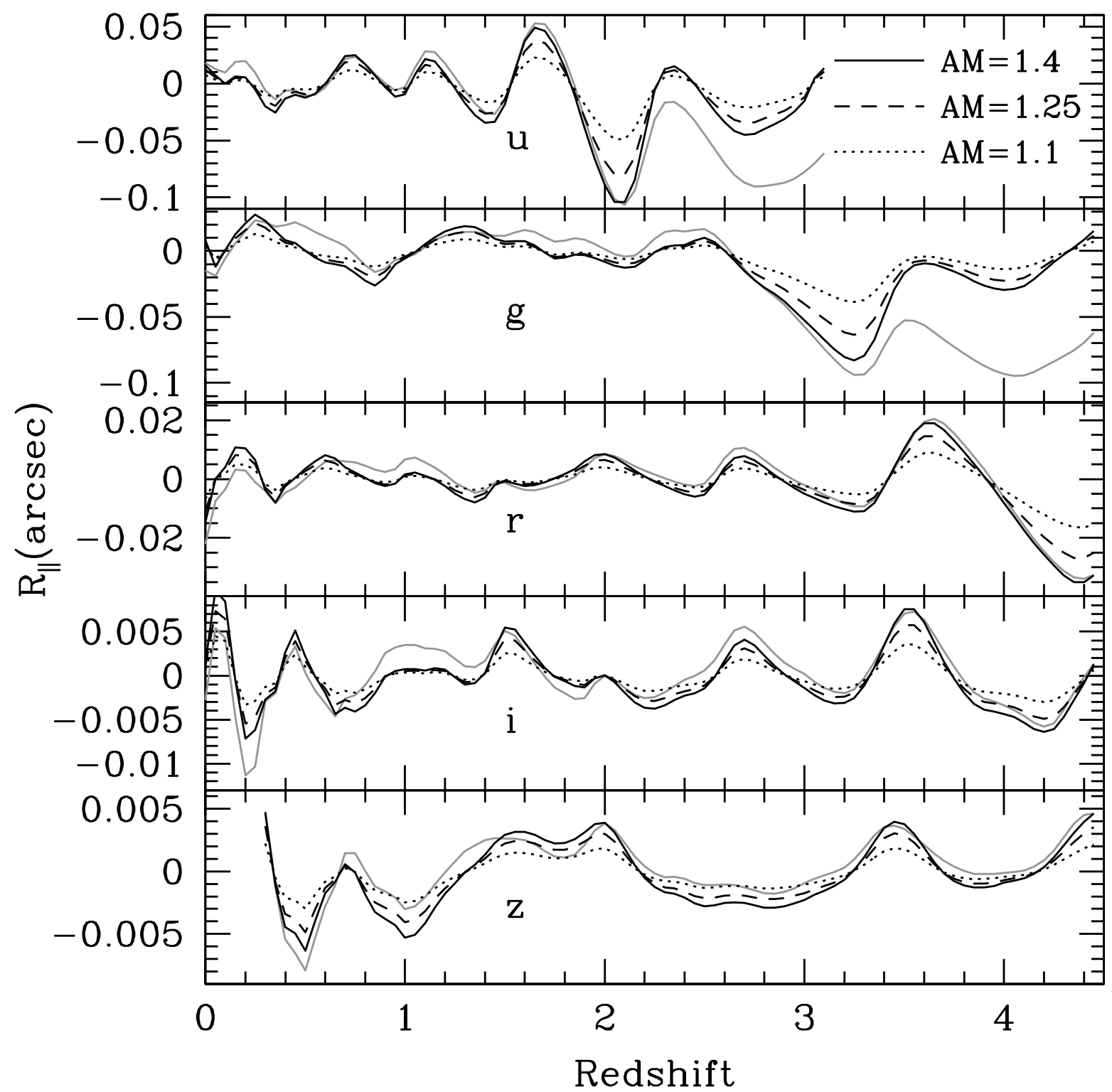

Fig. 4.- Expected change of quasar position as a function of redshift and bandpass. Each panel shows one of the ugriz filters and its expected positional change with respect to the position expected based on synthetic colors calculated using the Vanden Berk et al. (2001) SDSS composite quasar spectrum. Three airmass values are shown in black $(\mathrm{AM}=1.4$ : solid; $\mathrm{AM}=1.25$ : dashed; $\mathrm{AM}=1.1$ : dotted). The solid gray line is for $\mathrm{AM}=1.4$, but uses an $\alpha_{\nu}=-0.5$ power-law continuum instead of the synthetic colors to model the expected DCR. 

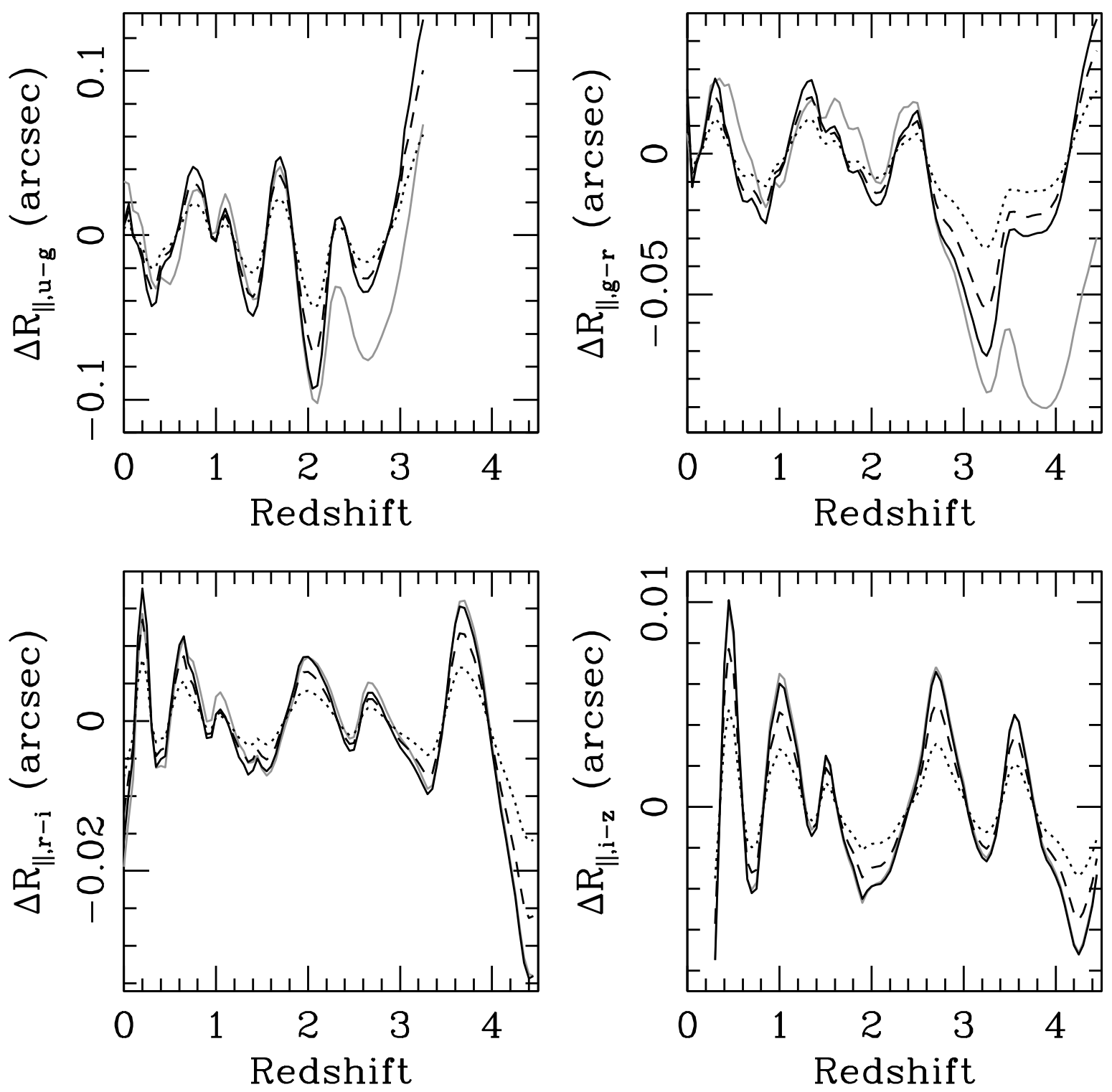

Fig. 5.- Predicted positional differences (in arcseconds) between adjacent bandpasses as shown in Fig. 4, again as a function of redshift and airmass. The difference in positional offsets is represented by $\Delta R_{\|, m-n}$ where $m$ and $n$ represent two bandpasses. For instance, $\Delta R_{\|, u-g}$ is the expected positional offset of the $u$ band with respect to the $g$ band. Line styles and colors are as in Fig. 4 . 


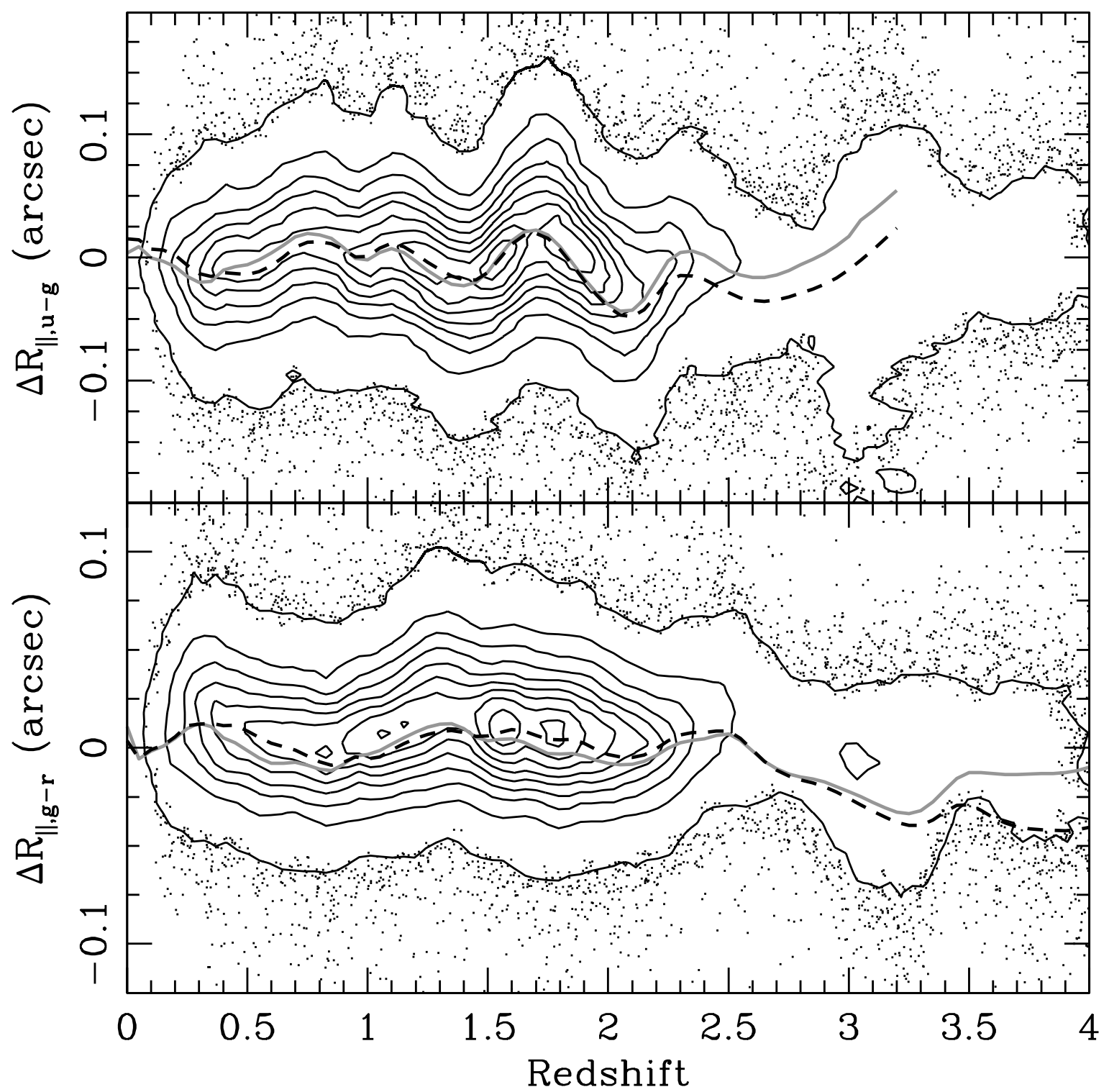

Fig. 6.- Observed positional offsets from emission line induced atmospheric dispersion for single-epoch observations of SDSS quasars. Only $\Delta R_{\|, u-g}$ and $\Delta R_{\|, g-r}$ are shown, since they are expected to show a measurable effect. The dashed line indicates the expected offsets from a quasar template assuming observations at $\mathrm{AM}=1.1$ and a color-based DCR correction; the solid gray line assumes a power-law model for the DCR correction. 


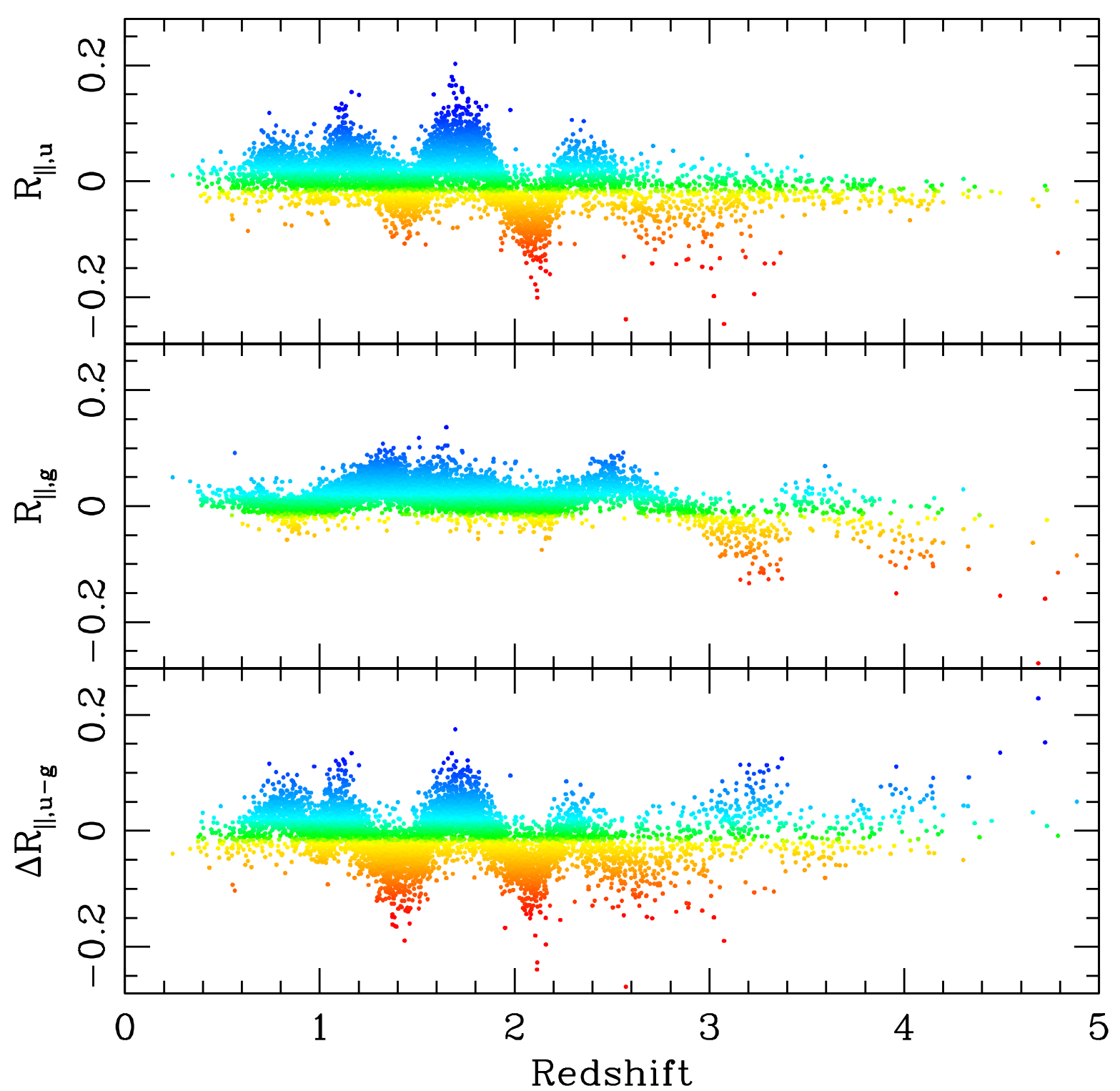

Fig. 7.- Astrometric offsets (in arcseconds) for combined, multi-epoch observations of 6430 SDSS quasars in stripe 82. Points are color-coded by the amount of their positional offsets along the parallactic angle. Bluer effective wavelengths lead to more positive offsets and are given by bluer points. The top, middle, and bottom panels are for $u$-band offsets, $g$-band offset, and the difference of the two, respectively. 


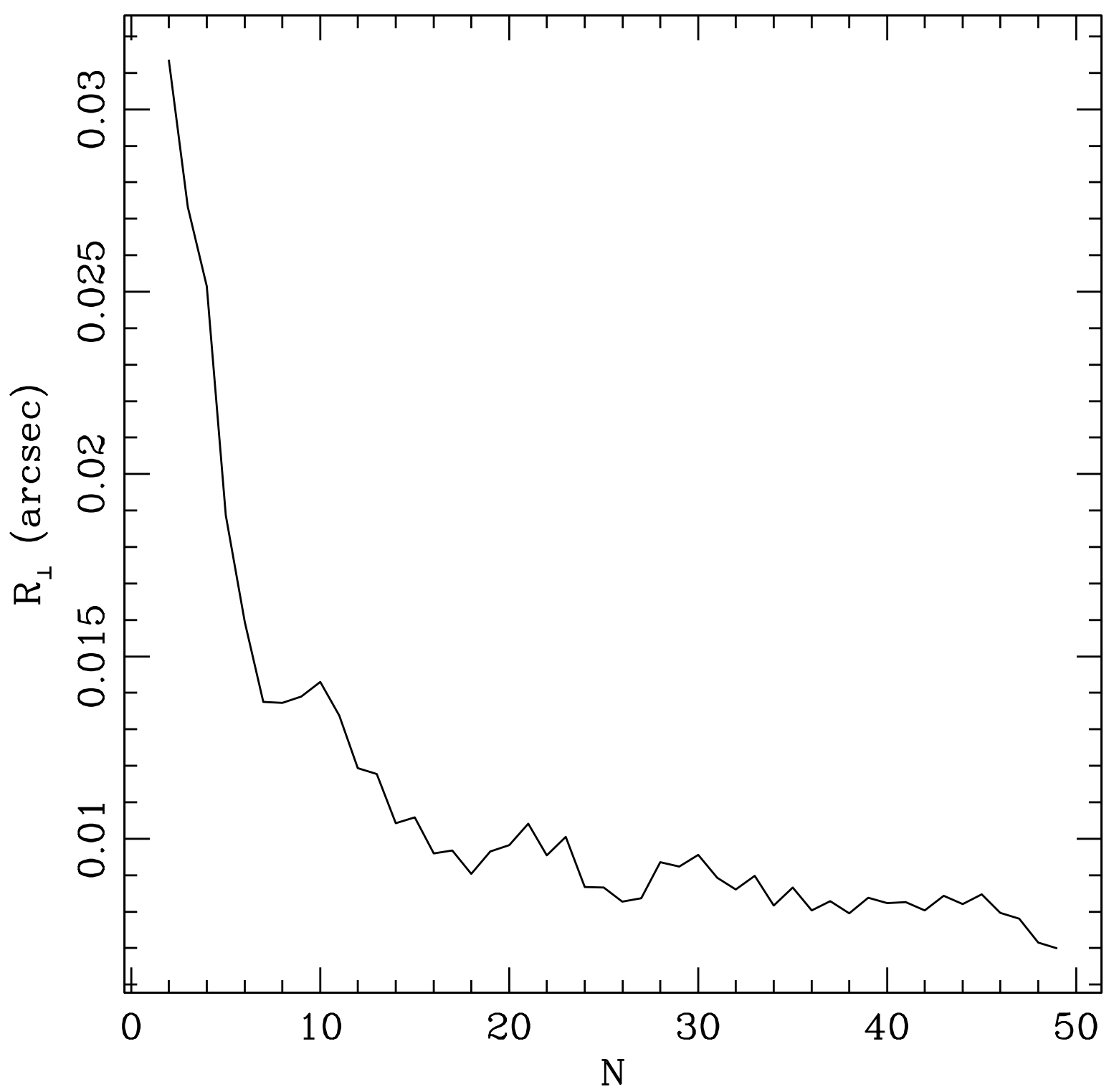

Fig. 8.- Reduction of random astrometric error as a function of number of combined epochs. Single epoch SDSS observations have 25-30 mas astrometric errors. Combining multiple observations can reduce this to $\sim 7-8$ mas. These results were derived from bootstrap resampling the deviations perpendicular to the parallactic angle for 50 white dwarf observations. 


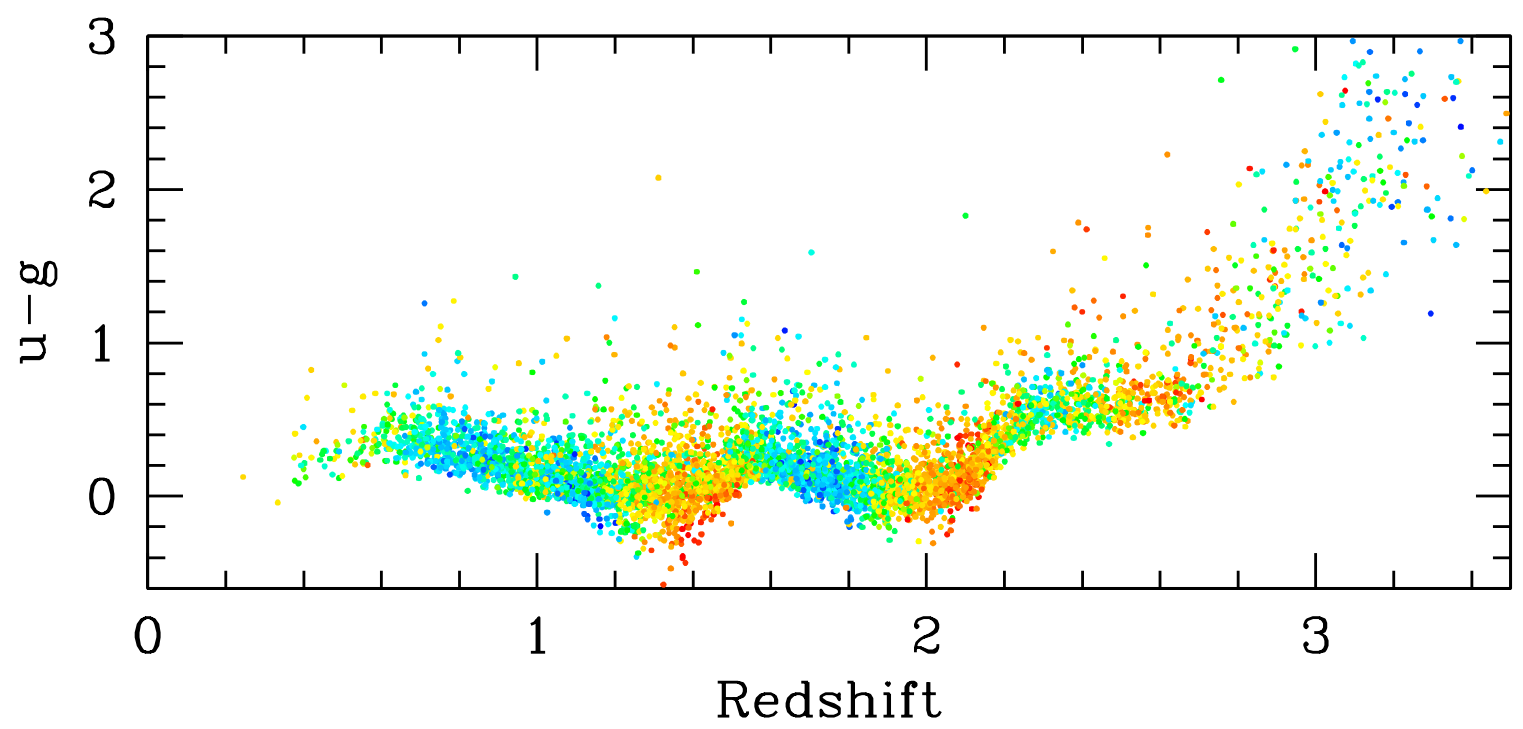

Fig. 9. $-u-g$ color as a function of redshift for SDSS quasars, color-coded by the positional offsets from Fig. 7. While an emission line moving through a bandpass produces a color change that is roughly symmetric with redshift, the positional offsets break this symmetry. 


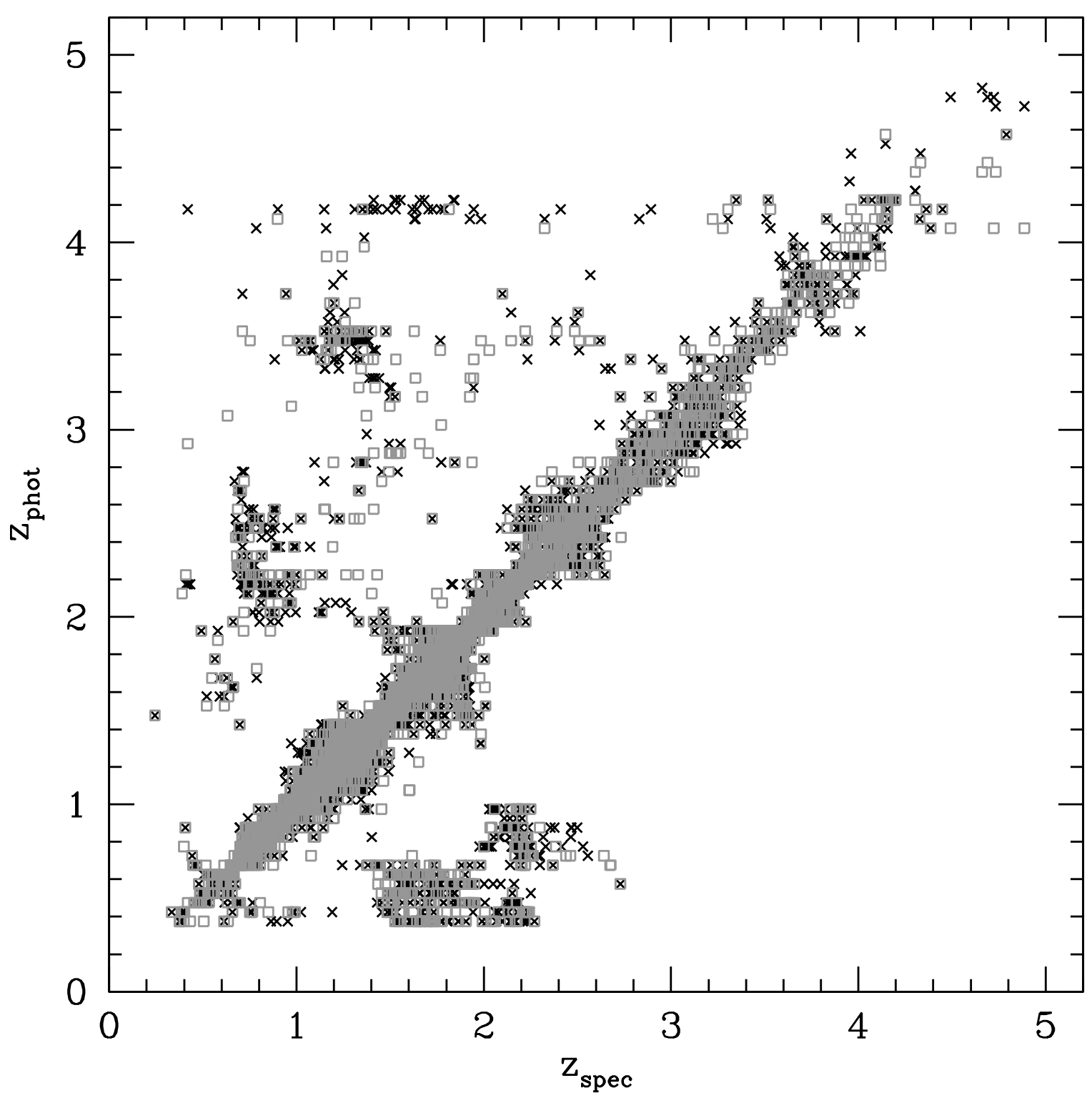

Fig. 10.- Comparison of spectroscopic and photometric redshifts. Black crosses show photo- $z$ only, whereas grey squares are estimates from photo- $z+$ astro- $z$. The latter have fewer catastrophic errors and a tighter distribution along the diagonal. 


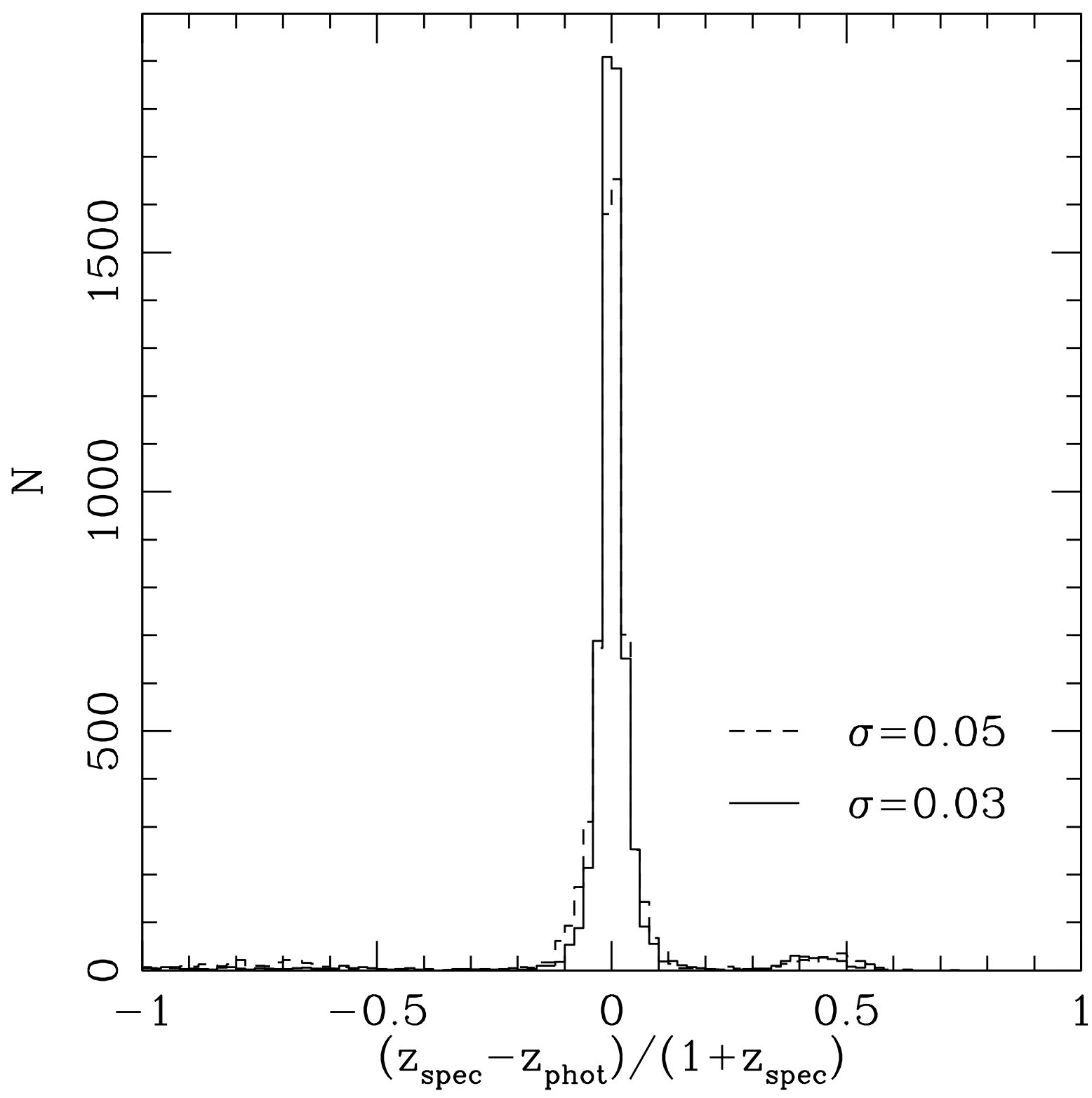

Fig. 11. - Fractional improvement in redshift estimates from the inclusion of astrometric offsets (solid) as compared to photometric redshifts from colors alone (dashed). Astro- $z$ information enables a significant improvement in accuracy. 


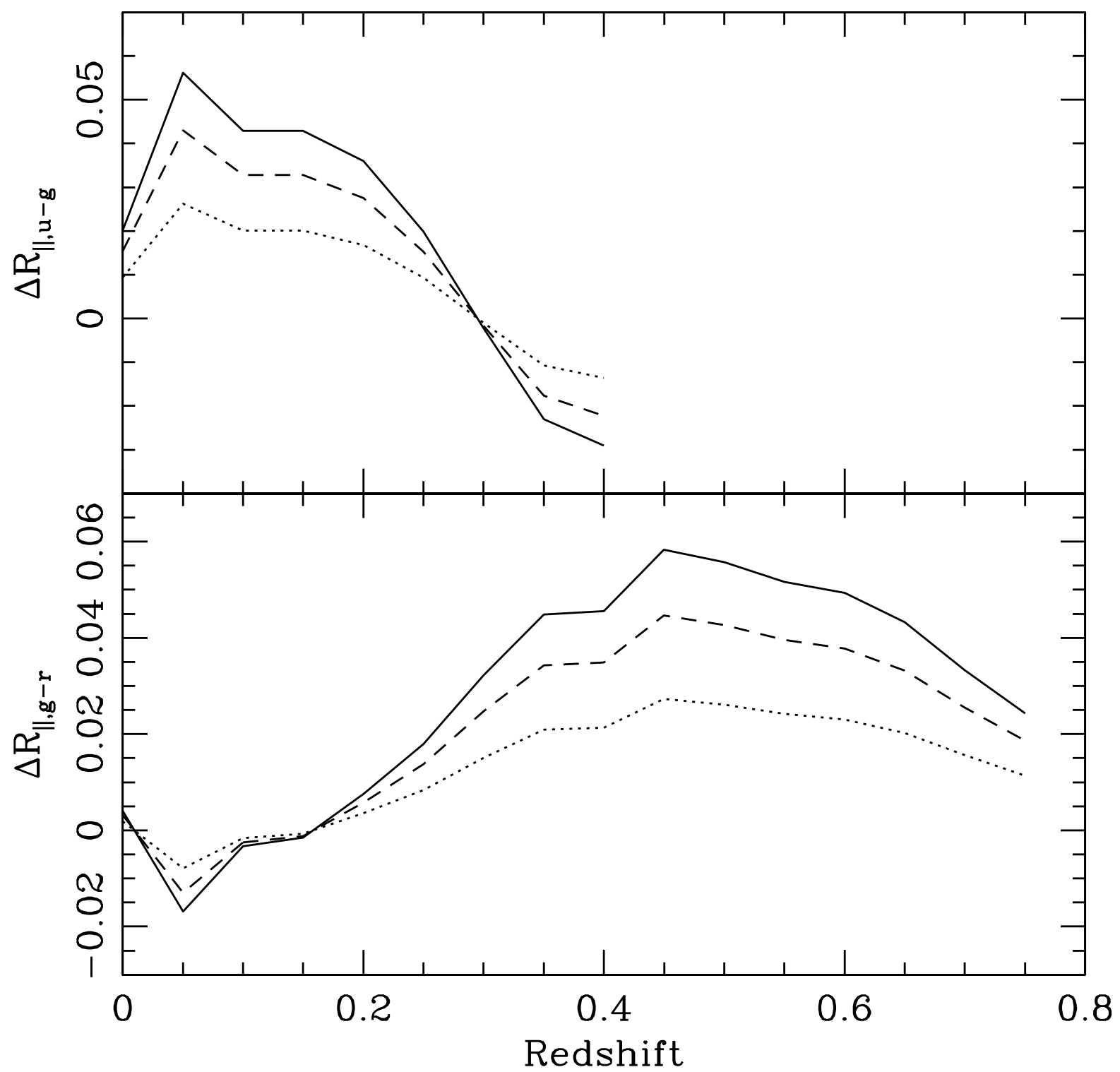

Fig. 12.- Positional offsets (in arcseconds) for type 2 quasars, based on a composite type 2 spectrum from Zakamska et al. (2003). Results for three airmasses are shown, labeled as in Fig. 4. While the offsets for type 2 quasars are smaller than for type 1 quasars, the falling trend in $R_{\|, u-g}$ coupled with the rising trend in $R_{\|, g-r}$ is encouraging with regard to type 2 redshift prediction. 


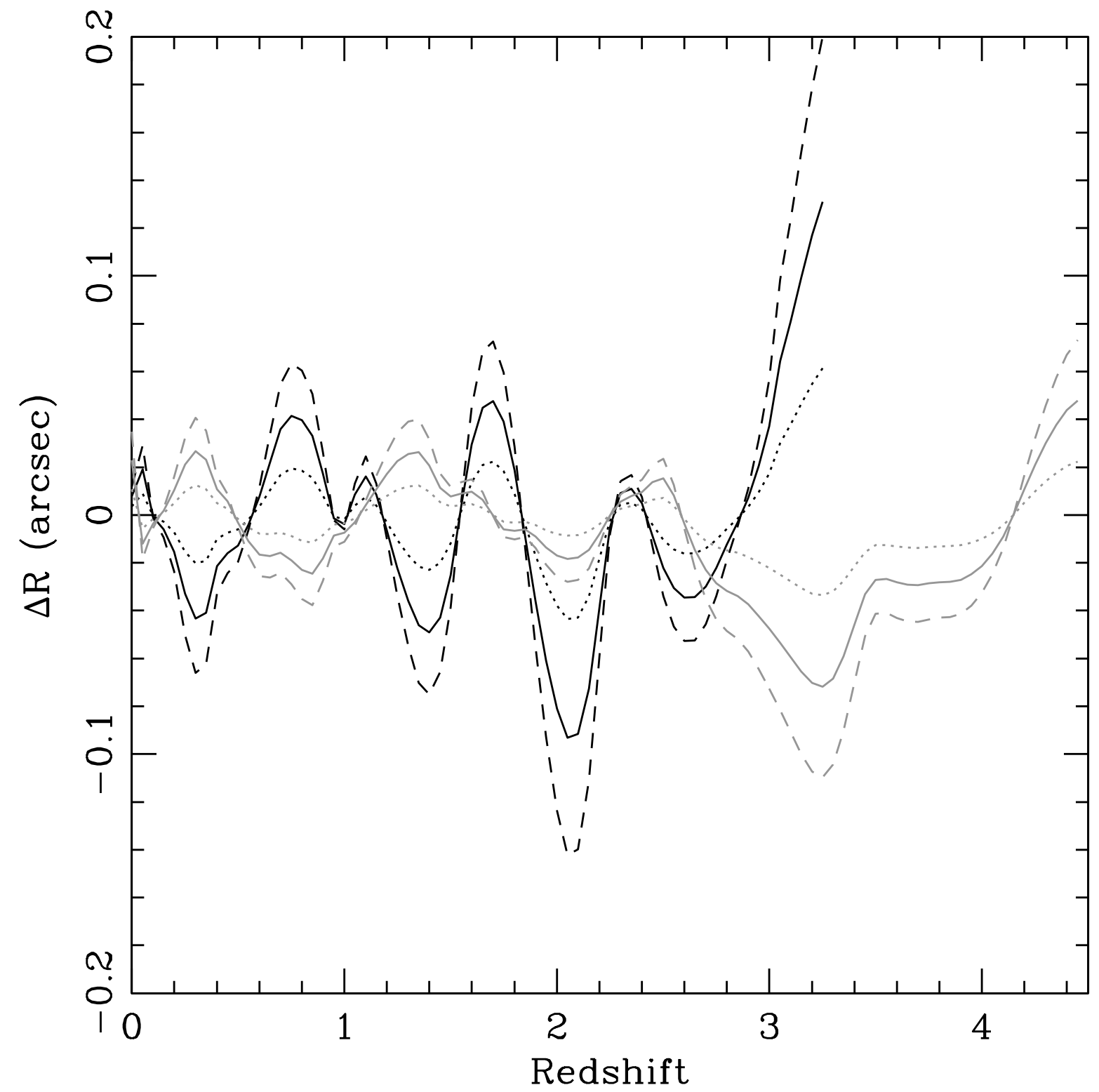

Fig. 13.- Positional offsets as a function of quasar redshift. $\Delta R_{\|, u-g}$ is shown in black, $\Delta R_{\|, g-r}$ in gray. Solid lines give $\mathrm{AM}=1.4$, dashed lines are $\mathrm{AM}=1.8$, and dotted lines are $\mathrm{AM}=1.1$. It is clear that $\mathrm{AM}=1.1$ does not provide significant leverage in resolving quasar redshift degeneracies, but nor is there significant gain in going from $\mathrm{AM}=1.4$ to $\mathrm{AM}=1.8$, suggesting moderate airmass observations. 